\title{
Tratamiento artroscópico de las fracturas de tercio distal de clavícula tipo 2 de Neer con sistema ZipTight
}

\author{
DOI: http://dx.doi.org//10.37315/SOTOCAV201928054149
}

Autor: CAÑETE SAN PASTOR P.

SERVICIO COT. HOSPITAL DE MANISES. CLÍNICA GASTALDI. VALENCIA

\begin{abstract}
Resumen.
OBJETIVO: Las fracturas de tipo II de Neer del tercio externo de la clavícula son fracturas con una alta tasa de falta de unión o unión defectuosa (hasta $30 \%$ ). Se han descrito múltiples técnicas quirúrgicas para su tratamiento. Además, varias técnicas artroscópicas. MÉTODOS. Hemos revisado retrospectivamente 31 casos con fracturas de tercio distal de la clavícula distal de Neer tipo II tratadas fijación coracoclavicular con un sistema de suspensión, con técnica artroscópica. RESULTADOS. Todas las fracturas, excepto 1, se han consolidado por completo en 3 meses, sin las desventajas de otros sistemas como Agujas de Kirschner, tornillos o placas. La tasa de consolidación ha sido del 96,8\% (todos menos 1 paciente). La movilidad obtenida fue muy satisfactoria: flexión de $176^{\circ}$, abducción de $145^{\circ}$, rotación externa de $80^{\circ}$, rotación interna con la mano hasta T11 y aducción horizontal de $132^{\circ}$. Los resultados funcionales obtenidos han sido excelentes en la mayoría de los pacientes: obteniendo 96 puntos en la escala de Constant y excelente UCLA en 28 casos. Este es uno de los estudios con más pacientes tratados con este tipo de técnica. Todos los pacientes han sido seguidos hasta la consolidación clínica y radiográfica de las fracturas. También aportamos algunas modificaciones técnicas a la técnica inicial que hemos estado obteniendo con la experiencia para mejorar el resultado clínico y radiológico de los pacientes. CONCLUSIÓN: El tratamiento artroscópico asistido de fracturas del tercio distal de clavícula Neer tipo II con un sistema Ziptight es una técnica reproducible que nos permite reducir y estabilizar la fractura y también diagnosticar y tratar posibles lesiones glenohumerales o del manguito rotador, obteniendo excelentes resultados clínicos y radiológicos.
\end{abstract}

Palabras clave: Fracturas clavícula, tratamiento artroscópico, ZipTight

\section{Summary.}

PURPOSE.Neer type II fractures of the external third of the clavicle are fractures with a high rate of nonunion or malunion (up to $30 \%$ ). Multiple surgical techniques have been describe their treatment. Also, several arthroscopic techniques. METHODS.We have retrospectively reviewed 29 cases with Neer type II distal third clavicle fractures treated with coracoclavicular fixation with a suspension system. RESULTS.All fractures except 1 have undergone complete consolidation in 3 months, without the disadvantages of other systems such as KW,screws or plates. The rate of consolidation has been $96.5 \%$ (all but 1 patient). The mobility obtained was very satisfactory: flexion of $176^{\circ}$, abduction of $145^{\circ}$, an external rotation of $80^{\circ}$, an internal rotation with the hand until T11 and horizontal adduction of $132^{\circ}$. The functional results obtained have been excellent in most patients: obtaining 96 points on the Constant scale and excellent UCLA in 26 cases. This is one of the studies with more patients treated with this type of technique. All patients have been followed until the clinical and radiographic consolidation of the fractures. We also contribute some technical modifications to the initial technique that we have been obtaining with the experience to improve the clinical and radiological result of the patients. CONCLUSION.The arthroscopic assisted treatment of Neer type II distal third clavicle fractures with a Ziptight system is a reproducible technique that allows us to reduce and stabilize the fracture and also to diagnose and treat possible glenohumeral o rotator cuff injuries, obtaining excellent clinical and radiological results.

\section{Correspondencia:}

Pablo Cañete San Pastor pablotrauma@gmail.com 
CAÑETE P. Tratamiento artroscópico de las fracturas de tercio distal de clavícula tipo 2 de Neer con sistema ZipTight.

\section{Introducción}

Las fracturas de clavícula son lesiones frecuentes, que suponen entre el 35 y $45 \%$ de las fracturas del hombro. Dentro de las fracturas de clavícula, las fracturas de tercio externo suponen el $15-25 \%$ del total. Neer clasificó las fracturas de tercio externo de clavícula en tres tipos: Tipo I lateral a los ligamentos coracoclaviculares; Tipo II medial a los ligamentos coracoclaviculares; y Tipo III fracturas que afectan a la articulación acromioclavicular. Las fracturas tipo II, se subclasifican en IIA y IIB: en la IIA los ligamentos coracoclaviculares permanecen insertados en el fragmento lateral, y en la IIB el ligamento conoide (el más medial de los ligamentos coracoclaviculares) está roto y el ligamento trapezoide (el más lateral) permanece insertado en el fragmento lateral. En las fracturas tipo II, especialmente en las tipo IIB, el peso del brazo desplaza el fragmento lateral hacia distal y el trapecio desplaza el fragmento medial hacia proximal. Este hecho explica la separación de ambos fragmentos óseos y que sean fracturas con un alta tasa de pseudoartrosis (hasta del $30 \%)^{1-4}$.

Se han descrito numerosas técnicas quirúrgicas para el tratamiento de estas fracturas: fijación percutánea con aguas de kirschner, tornillos coracoclaviculares, cerclajes alámbricos, reducción abierta y fijación interna con placas gancho subacromiales o placas preconformadas de tercio externo de clavícula y reconstrucción de los ligamentos coracoclaviculares extirpando o no el fragmento lateral ${ }^{5-11}$. Se han descrito varias técnicas artroscópicas para el tratamiento de estas fracturas ${ }^{12-15}$.

En este estudio hemos revisado de forma retrospectiva 31 casos con fracturas de tercio externo de clavícula tipo II de Neer tratadas artroscópicamente con fijación coracoclavicular con un sistema de suspensión. Todos las fracturas excepto 2 han presentado una consolidación completa en $3^{\prime} 5$ meses, sin los inconvenientes de otros sistemas de fijación como AK, tornillos o placas.

\section{Material y Métodos}

Hemos revisado 31 pacientes con fracturas desplazadas de tercio distal de clavícula tipo IIB en la clasificación de Neer, tratados con fijación coracoclavicular artroscópica con sistema Ziptight (Zimmer-Biomet, Warsaw, Indiana) y seguidos hasta la consolidación clínica y radiográfica de la fractura. Hemos estudiado retrospectivamente las radiografías, la evolución de los pacientes con la escala de Constant, y las complicaciones aparecidas.

\section{TÉCNICA}

Todos los pacientes fueron intervenidos con anestesia general más plexular. Colocamos al paciente en posición de silla de playa. Utilizamos tracción longitudinal, bien con sistema de fijación al brazo y $5 \mathrm{~kg}$ de peso; o bien con un posicionador neumático Trimano que nos permite traccionar el brazo y fijarlo en esa posición. Esta tracción la mantendremos en el proceso de visualización de la coracoides y colocación del dispositivo de fijación y la liberaremos en el momento en que vayamos a reducir y fijar la fractura.

Creamos el portal posterior estándar y entramos en la articulación glenohumeral para descartar la presencia de lesiones asociadas (labrum, manguito, lesiones condrales). Posteriormente accedemos al espacio subacromial y localizamos el ligamento coracoacromial que será nuestra guía para localizar la coracoides. Con la óptica en el portal posterior, creamos un portal lateral, justo en el borde anterior del acromion. Con el sinoviotomo y el vaporizador iremos limpiando el tejido bursal y localizando el ligamento coracoacromial, que seguiremos desde el borde anterior del acromion hasta la coracoides. Una vez localizada la coracoides, cambiaremos la óptica al portal lateral y crearemos otro portal anterior, ayudados con una aguja, con la técnica de fuera a dentro. Limpiaremos bien la base de la coracoides, para tener una buena visión.

Con la óptica en el portal lateral, introduciremos una guía de reconstrucción del ligamento cruzado anterior modificada para poner la angulación deseada (Fig. 1). Apoyaremos la guía en la parte inferior de la base de la coracoides, centrada en el plano mediolateral, para evitar fracturas al pasar la broca.

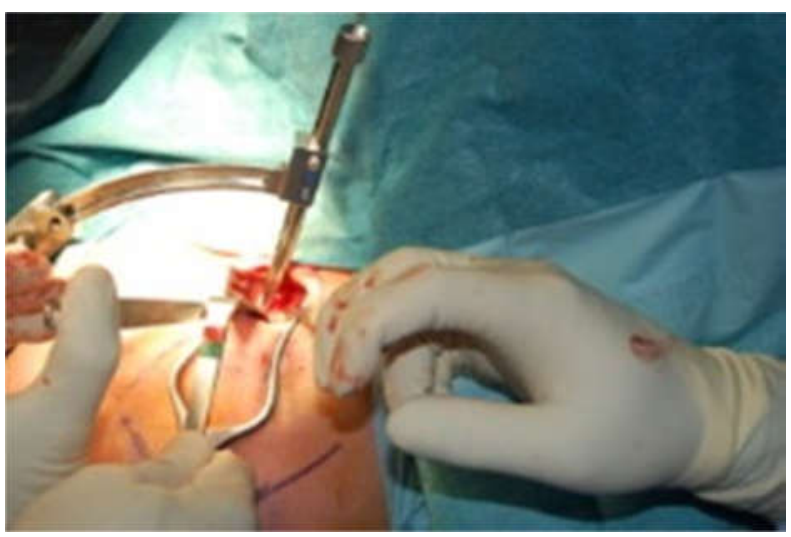

Figura 1: Guía modificada del ligamento cruzado anterior apoyada en la clavícula.

Pasaremos ahora a una parte de cirugía abierta, realizando una incisión de unos $3 \mathrm{~cm}$, siguiendo las líneas de Langer para exponer la fractura del tercio distal de la clavícula.

Con la óptica en el portal lateral, comprobamos la correcta colocación del pie de la guía en la base de la coracoides, y apoyaremos la guía de brocado en la diáfisis de la clavícula, al menos $1,5 \mathrm{~cm}$ medial a la fractura. En este momento soltamos la tracción del hombro, para facilitar la reducción de la fractura. La misma guía tiene un sistema de bloqueo que facilita la reducción de la fractura en el plano superoinferior. Con palpación manual e inspección comprobamos que no exista desplazamiento de la fractura en el plano anteroposterior. Podemos inclinar la guía para conseguir una entrada posteroanterior desde superior a inferior, llevando así el fragmento medial hacia inferior y 
CAÑETE P. Tratamiento artroscópico de las fracturas de tercio distal de clavícula tipo 2 de Neer con sistema ZipTight.

anterior (que son las direcciones habituales del desplazamiento)

Un pin a través de la guía, que atravesará las dos corticales de la clavícula y las dos corticales de la coracoides. Comprobamos la correcta posición, centrada en clavícula y coracoides, del pin. A través del pin, usamos una broca canulada de 4'5 mm (Fig. 2), para atravesar las 4 corticales de nuevo. Retiramos el pin y a través de la broca canulada pasamos una sutura transportadora desde la clavícula hasta la coracoides y la recuperamos por el portal anterior (Fig. 3). Utilizamos esta sutura para pasar el implante desde la clavícula hasta la coracoides. Comprobamos con visión artroscópica el correcto asentamiento de el botón metálico en la base de la coracoides, y colocamos el otro botón las suturas del extremo clavicular del implante (Fig. 4).

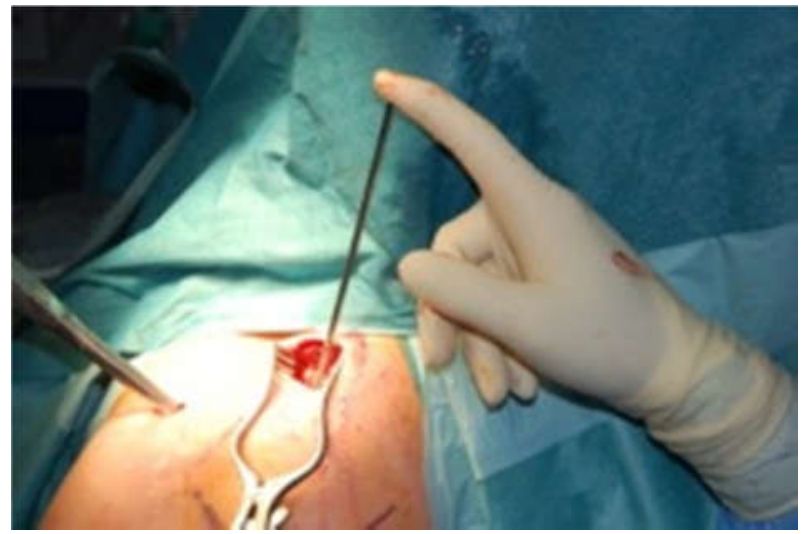

Figura 2: Broca canulada $4,5 \mathrm{~mm}$ que pasa las dos corticales de la clavícula y las dos de la apófisis coracoides.

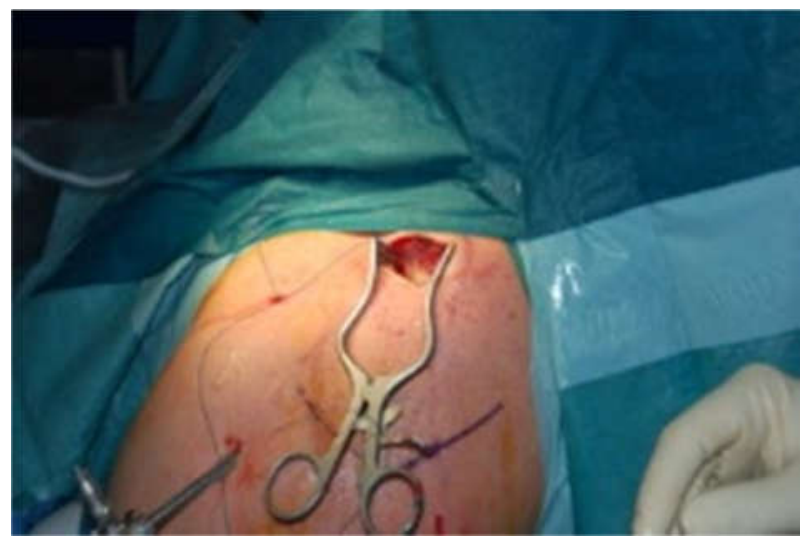

Figura 3: Sutura transportadora desde la clavícula hasta la coracoides, recuperada a través del portal anterior. Óptica en el portal lateral.

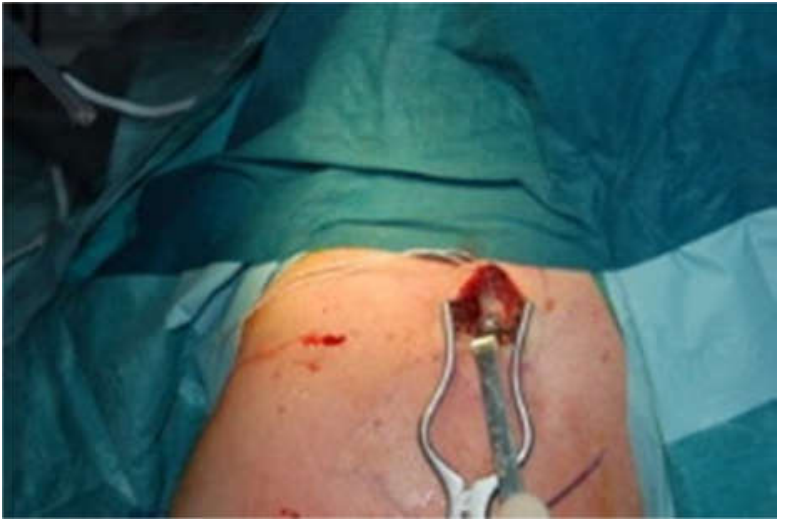

Figura 4: Botón apoyado en la clavícula y bloqueando las suturas, manteniendo la reducción de la fractura.

El ziptight es un implante autobloqueante, con el sistema ziploop, que al traccionar de las suturas deslizantes, se reduce el resto de bucles y conseguimos una reducción de la clavícula sobre la coracoides, reduciéndose así la fractura en el plano superoanterior (y si también algo de reducción en el anteroposterior si hemos inclinado ligeramente la guía al hacer los orificios). Podemos ayudar a la reducción comprimiendo en dirección anteroinferior el fragmento medial y empujando del brazo hacia arriba para subir el fragmento distal. El sistema es autobloqueante y no requiere de nudos, que podrían producir molestias posteriores.

Comprobamos la reducción clínica y radiológicamente. Es importante el correcto cierre de la fascia deltotrapezoidea (Fig. 5).

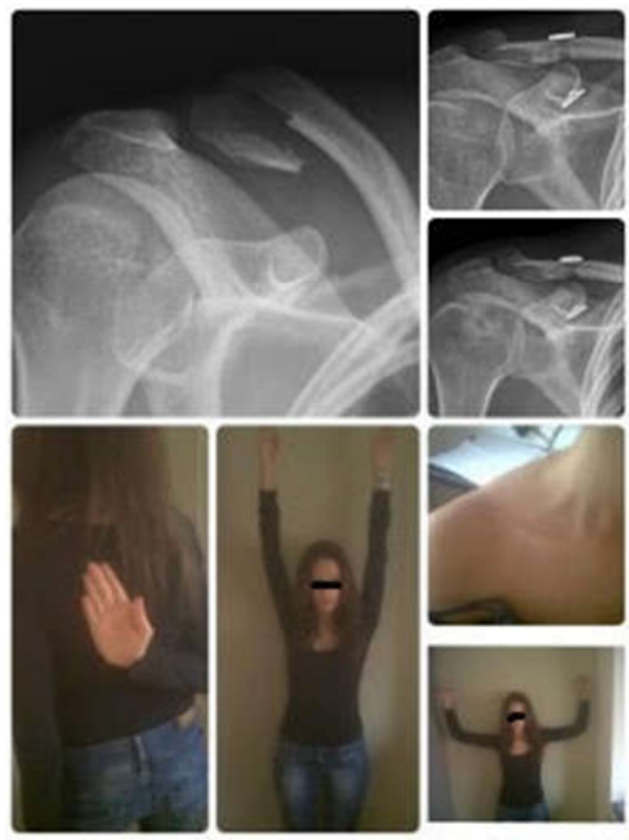

Figura 5: 1. Radiografía de fractura aguda. 2. Control de radiografía postoperatorio inmediato. 3 . Control de rayos x 3 meses con la fractura curada. 4,5,6 y 7. Control clínico a las 3 meses que muestra el excelente resultado funcional y estético. 
CAÑETE P. Tratamiento artroscópico de las fracturas de tercio distal de clavícula tipo 2 de Neer con sistema ZipTight.

Colocamos un sling standard al paciente durante 6 semanas. Se puede retirar el cabestrillo para el aseo personal. Comenzar los ejericios pasivos y autoasistidos a las 3 semanas y los activos a las 6 semanas. No permitimos cargar peso, ni realizar esfuerzos ni actividades deportivas hasta comprobar la consolidación de la fractura (unos 3 meses).

En este punto, añadimos una nota técnica. Tras haber tenido un caso de retardo de consolidación por falta de reducción anteroposterior de la fractura (la fractura finalmente consolidó a los 12 meses (Fig. 6)), añadimos un sistema de reducción y fijación de la fractura en el plano anterosuperior (en un caso con tornillos de compresión con mal resultado: la fractura no consolidó y requirió una nueva cirugía para añadir injerto y fijación con placa (Fig. 7). Hemos modificado la técnica y añadimos un cerclaje interfragmentario en 8 con sutura de alta resistencia, como ya ha sido descrito por otros autores (Fig. 8). Añadiendo este cerclaje, obtenemos mejor estabilidad y curación más temprana de la fractura. Todos estos casos (más de 20 casos) con variaciones técnicas no están incluídos en el estudio.
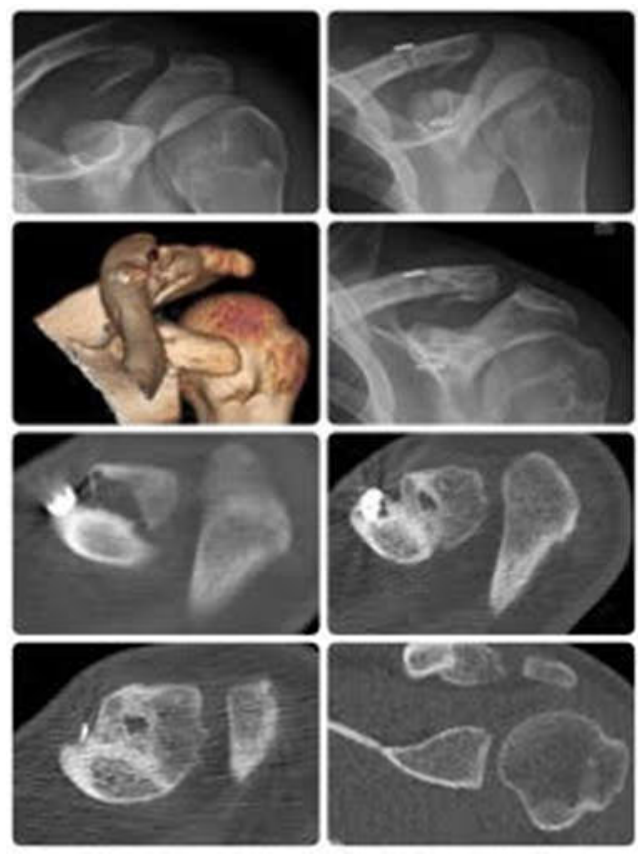

Figura 6: Fractura aguda 1.Radiografía simple de la clavícula. 2. Control inmediato de rayos $\mathrm{x}$ postoperatorio. 3. TAC después de 6 meses. 4 . Radiografía después de 6 meses. 5.TAC después de 6 meses. 6,7 y 8 . TAC después de 12 meses.

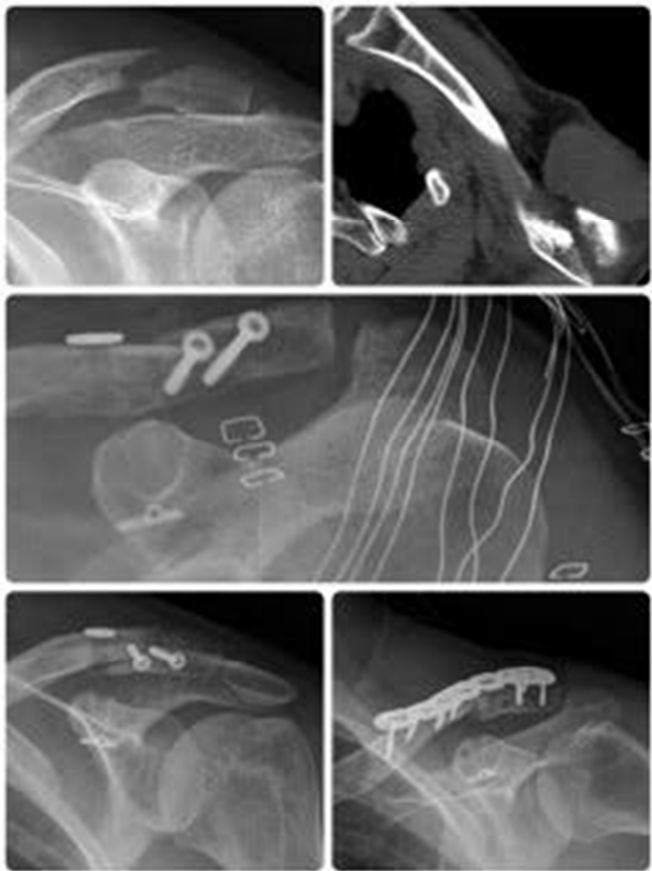

Figura 7: 1. Radiografía de fractura aguda. 2.TAC fractura aguda con el desplazamiento en la dirección anteroposterior. 3. Control inmediato de la radiografía postoperatoria con el cierre hermético y dos tornillos de compresión anteroposterior. 4. Control de rayos $X$ después de 10 meses sin unión. 5. Resultado final con injerto de placa y hueso.

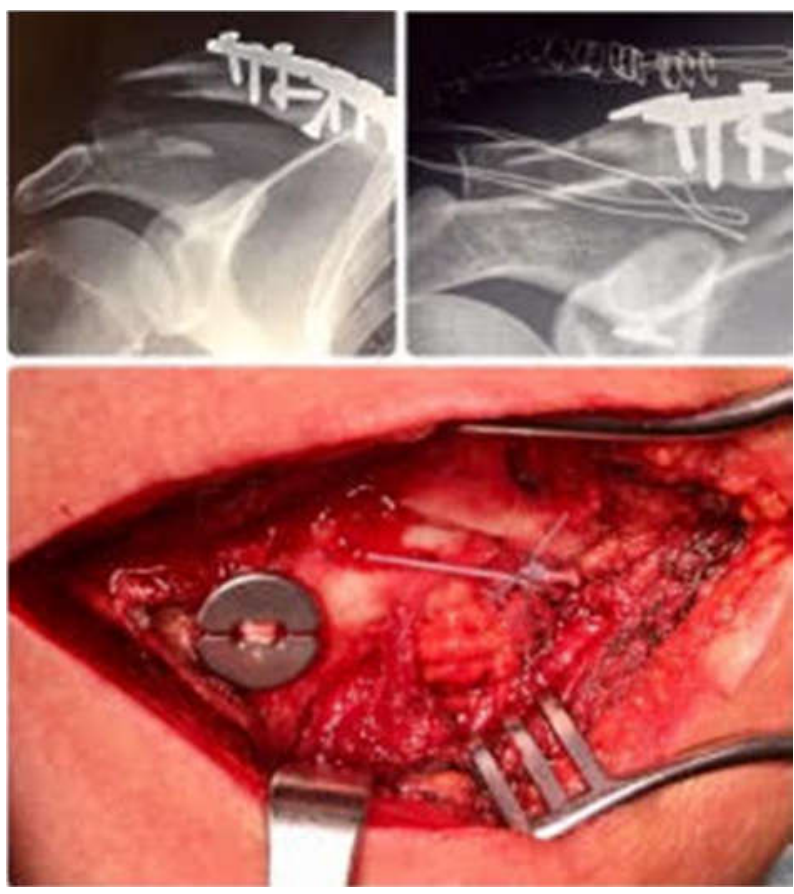

Figura 8: Imagen del botón en la clavícula y el cerclaje interfragmentario con suturas de alta resistencia para reducir la fractura en la dirección anteroposterior y aumentar la estabilidad de la reducción. 


\section{Resultados}

Hemos revisado 31 pacientes, 23 hombres y 8 mujeres. La edad media era de 35,2 años (18-59). Se han realizado controles radiográficos en el postoperatorio inmediato, a las 2-3 semanas, 6-8 semanas, a los 3 meses y hasta la consolidación. La tasa de consolidación $96,8 \%$ (todos menos 1 paciente)

La movilidad media obtenida ha sido muy satisfactoria con una Flexión anterior de $176^{\circ}$, una abducción de $145^{\circ}$, una rotación externa de $80^{\circ}$, una rotación interna con la mano hasta T11 y una adducción horizontal de $132^{\circ}$.

Los resultados funcionales obtenidos has sido excelentes en la mayoría de los pacientes. Hemos evaluado la función de los pacientes con las escalas de Constant y UCLA. Obteniendo 96 puntos en la escala de Constant y UCLA excelente en 28 casos, con recuperación completa a los 3 meses

Todos los pacientes excepto dos consiguieron la consolidación radiográfica de la fractura a los 3 meses: el paciente presentó un retardo de consolidación, produciéndose la curación de la fractura a los 12 meses, lo que verificamos con un TAC (Fig. 6).

Hemos obtenido complicaciones en dos casos:

Un caso de intolerancia al material, con infección subclínica que se resolvió tras la retirada del material a los 6 meses, tras la consolidación de la fractura.

Otro paciente con mal resultado a pesar de la correcta consolidación de la fractura y la recuperación completa de la movilidad. Precisó de una segunda intervención en la que se realizó extracción del material, resección del tercio distal de la clavícula y plicatura capsular anterior por microinestabilidad. Posteriormente desarrolló una discinesia escapular. Es el único caso laboral de la serie.

\section{Discusión}

Las fracturas tipo II de tercio distal de clavícula, en especial las tipo IIB son fracturas habitualmente desplazadas e inestables en las que un tratamiento no quirúrgico provoca altas tasas de malos resultados funcionales.

Se han descrito numerosas técnicas quirúrgicas para el tratamiento de estas fracturas, entre las que se incluyen la fijación asistida por artroscopia ${ }^{5-11}$.

El sistema ZipTight fue diseñado para la estabilización de las luxaciones acromioclaviculares. Hemos empleado este sistema para el tratamiento de este tipo de fracturas con excelentes resultados, consiguiendo una recuperación funcional completa del hombro. Ya se han publicado estudios con el uso de este tipo de implantes para las fracturas de tercio externo de clavícula con excelentes resultados ${ }^{12-15}$.
El uso de la artroscopia, además de ser una técnica mínimamente invasiva que respeta los tejidos y nos posibilita acceder a la base de la coracoides con una mínima disección, nos permite además realizar un diagnóstico y tratamiento de la patología articular glenohumeral o del manguito de los rotadores en caso de que existiera. (Burkhart habla de hasta el $40 \%$ de los casos en luxaciones acromioclaviculares) ${ }^{16-22}$.

El Ziptight es un sistema rápido y seguro, autobloqueante, que nos ayuda a reducir y mantener la reducción, y que no precisa de nudos, evitando los inconvenientes de los nudos con sutura de alta resistencia que pueden aparecer, ocasionando irritación y molestias a los pacientes.

Es conveniente tener un control con radioscopia en quirófano, al menos en los primeros casos, aunque según se gana experiencia, no lo consideramos fundamental.

Es importante controlar la reducción en el plano anteroposterior, ya que el sistema coracoclavicular nos reduce la fractura en el plano superoinferior, pero no anteroposterior. Si tenemos un desplazamiento importante en este plano, podemos tener retardos o incluso ausencias de consolidación.

En algunos casos colocamos la guía ligeramente más posterior en la diáfisis de la clavícula y reducimos la fractura en ambos planos ayudados de un periostotomo antes de realizar las perforaciones. Con esto conseguimos una mejor reducción y fijación de la fractura en el plano anteroposterior. También hemos añadido en 1 caso (no incluido en el estudio) tornillos anteroposterior interfragmentarios (Fig. 7). Aunque actualmente preferimos añadir un cerclaje en 8 con sutura de alta resistencia entre los dos fragmentos de la clavícula, que además de estabilidad anteroposterior, proporciona compresión interfragmentaria (Fig. 8).

Aunque ningún paciente de esta serie (pacientes tratados sin cerclaje interfragmentario) ha desarrollado una seudoartrosis, consideramos que añadir el cerclaje aporta mejor reducción y mayor estabilidad de la fractura. No hemos comparado ambas técnicas.

Esta técnica nos proporciona un excelente resultado funcional, con una reducción estable evitando los inconvenientes de otros sistemas (migración de las agujas, intolerancia a las placas, obligación de retirar las placas gancho, osteolisis de tornillos coracoclaviculares, pérdida de reducción de agujas o placas, etc.). Además de proporcionar un buen resultado estético

Aunque hay varios estudios publicados sobre el tratamiento artroscópico de las fracturas de tercio externo de clavícula, este es uno de los estudios con más pacientes tratados con este tipo de técnica. Todos los pacientes han sido seguidos hasta la consolidación clínica y radiográfica de las fracturas. Aportamos además algunas modificaciones técnicas que con la experiencia hemos ido obteniendo para mejorar el resultado clínico y radiológico de los pacientes. 
CAÑETE P. Tratamiento artroscópico de las fracturas de tercio distal de clavícula tipo 2 de Neer con sistema ZipTight.

\section{Conclusiones}

En conclusión, el tratamiento de las fracturas de tercio externo de clavícula tipo II de Neer con sistema Ziptight de fijación coracoclavicular asistida por artroscopia es una técnica reproducible que nos permite una reducción y estabilización adecuada de la fractura, nos permite diagnosticar y tratar las posibles lesiones intraarticulares del hombro, y con ella obtenemos unos excelentes resultados clínicos y radiológicos. 
CAÑETE P. Tratamiento artroscópico de las fracturas de tercio distal de clavícula tipo 2 de Neer con sistema ZipTight.

\section{Bibliografía}

1. Neer CS. Nonunion of the clavicle. JAMA 1960;172:1006- 1011.

2. Neer CS II. Fractures of the clavicle. In: Rockwood CA Jr, Green DP, eds. Fractures in Adults. Ed 2. Philadelphia: JB Lippincott, 1984; 707-713.

3. Neer CS II. Fractures of the distal third of the clavicle. Clin Orthop 1968;58:43-50.

4. Neer CS II. Fracture of the distal clavicle with detachment of the coraco-clavicular ligaments in adults. J Trauma 1963;3: 99-110.

5. Moore TO. Internal pin fixation for fracture of the clavicle. Am Surg 1951;17:580-583.

6. Mullaji AB, Jupiter JB. Low-contact dynamic compression plating of the clavicle. Injury 1994;25:41-45.

7. Edwards DJ, Kavanagh TG, Flannery MC. Fractures of the distal clavicle: A case for fixation. Injury 1992;23:4446.

8. Van der Meijden O.A., Gaskill T.R., Millett P.J. Treatment of clavicle fractures: Current concepts review. J Shoulder Elbow Surg. 2012;21:423-429.

9. Kona J, Bosse MJ, Staeheli JW, Rosseau RL. Type II distal clavicle fractures: A retrospective review of surgical treatment. J Orthop Trauma 1990;4:115-120.

10. Levy O. Simple, minimally invasive surgical technique for treatment of type 2 fractures of the distal clavicle. $J$ Shoulder Elbow Surg 2003;12:24-28.

11. Ballmer FT, Gerber C. Coracoclavicular screw fixation for unstable fractures of the distal clavicle. A report of five cases. J Bone Joint Surg Br 1991;73:291-294.

12. Geoffroy Nourissat, M.D., Carlos Kakuda, M.D., Christian Dumontier, M.D., Alain Sautet, M.D., and Levon Doursounian, M.D. Arthroscopic Stabilization of Neer Type 2 Fracture of the Distal Part of the Clavicle. Artrhoscopy 2007;23:674.e1-674.e4

13. Luis Natera Cisneros, M.D., Juan Sarasquete Reiriz, M.D. Arthroscopic-Assisted Management of Unstable DistalThird Clavicle Fractures: Conoid Ligament Reconstruction and Fracture Cerclage With Sutures. Arthrosc Tech. 2015 Dec; 4(6): e655-e661

14. Pujol N., Desmoineaux P., Boisrenoult P., Beaufils P. Arthroscopic treatment of comminuted distal clavicle fractures (Latarjet fractures) using 2 double-button devices. Arthrosc Tech. 2013;2:e61-e63.

15. Checchia S.L., Doneux P.S., Miyazaki A.N., Fregoneze M., Silva L.A. Treatment of distal clavicle fractures using an arthroscopic technique. J Shoulder Elbow Surg. 2008;17:395-398.

16. Jerosch J., Filler T., Peuker E., Greig M., Siewering U. Which stabilization technique corrects anatomy best in patients with AC-separation? An experimental study. Knee Surg Sports Traumatol Arthrosc. 1999;7:365-372.

17. Van der Meijden O.A., Gaskill T.R., Millett P.J. Treatment of clavicle fractures: Current concepts review. J Shoulder Elbow Surg. 2012;21:423-429.

18. Madsen W., Yaseen Z., LaFrance R. Addition of a suture anchor for coracoclavicular fixation to a superior locking plate improves stability of type IIB distal clavicle fractures. Arthroscopy. 2013;29:998-1004.

19. Hanflik A., Hanypsiak B.T., Greenspoon J., Friedman D.J. Open reduction internal fixation of distal clavicle fracture with supplementary button coracoclavicular fixation. Arthrosc Tech. 2014;3:551-554.

20. Levy O. Simple, minimally invasive surgical technique for treatment of type 2 fractures of the distal clavicle. J Shoulder Elbow Surg. 2003;12:24-28.

21. Shin S.J., Roh K.J., Kim J.O., Sohn H.S. Treatment of unstable distal clavicle fractures using two suture anchors and suture tension bands. Injury. 2009;40:1308-1312.

22. Paolo Arrigoni, M.D., Paul C. Brady, M.D., Leonardo Zottarelli, M.D., Johannes Barth, M.D., Pablo Narbona, M.D., David Huberty, M.D., Samuel S. Koo, M.D., Ph.D., Christopher R. Adams, M.D., Peter Parten, M.D., Patrick Denard, M.D., Stephen S. Burkhart, M.D. Associated Lesions Requiring Additional Surgical Treatment in Grade 3 Acromioclavicular Joint Dislocations Arthroscopy; 30:6-10. 